\title{
Pénuries de médicaments : Que peuvent faire les pharmaciens d'hôpitaux?
}

\author{
par Régis Vaillancourt
}

L es pharmaciens d'hôpitaux se sont habitués aux pénuries de médicaments au cours des dernières années. Par exemple, en 2010, 94 \% des pharmaciens canadiens sondés ont affirmé avoir eu des problèmes à trouver un médicament au cours de la semaine précédente ${ }^{1}$. Un sondage mené en 2010 auprès des membres de l'American Society of Health-System Pharmacists a révélé que les pharmaciens de même que les techniciens en pharmacie passaient en moyenne de 8 à 9 heures par semaine à résoudre des problèmes liés aux pénuries de médicaments ${ }^{2}$. De plus, ces pénuries ne sont pas limitées à l'Amérique du Nord; elles sont un problème mondial ${ }^{3}$. Leurs causes sont multiples, notamment ruptures d'approvisionnement, changements aux exigences réglementaires, pénuries de matières premières, retraits, stratégies gouvernementales d'établissement des prix et monopolisation de la fabrication ${ }^{1}$. Dans la foulée des pénuries actuelles des produits de Sandoz (déclarées en février 2012), les gestionnaires des pharmacies d'hôpitaux passent le plus clair de leur temps à surveiller l'approvisionnement en médicaments et à travailler avec les cliniciens afin de composer avec ces pénuries. La vraie question qu'on doit se poser ici est : que peuvent faire les pharmaciens d'hôpitaux pour prévenir l'exacerbation de cette crise d'origine humaine à laquelle nous faisons présentement face?

Dans cette ère de mondialisation, les fabricants de médicaments, tout comme les hôpitaux, cherchent continuellement à réduire les coûts. L'un des moyens pour y parvenir est la concentration de la fabrication. Or, lorsqu'un fabricant donné connaît un ralentissement de sa production, c'est toute la planète qui peut être touchée. Dans une récente étude publiée par la Food and Drug Administration américaine, 55 (43\%) des 127 pénuries ont été attribuées à des problèmes de qualité de fabrication ${ }^{4}$. Cela vous dit quelque chose?

Quel rôle ont donc joué les pharmaciens d'hôpitaux dans la genèse de la situation que nous connaissons présentement? Les hôpitaux ont pour mandat d'assurer des soins de haute qualité tout en faisant des économies. En tant que gestionnaires de pharmacie, nombreux sommes-nous à faire affaire avec des groupements d'achats, comme SigmaSanté au Québec et
Medbuy ou HealthPRO dans le reste du Canada. Ces trois groupements d'achats représentent environ $90 \%$ du marché des pharmacies d'hôpitaux canadiennes. Alors, quel est le lien entre les achats groupés et les pénuries actuelles de médicaments? Eh bien, il provient du concept du « meilleur offrant qui remporte tout $»^{3}$.

À l'heure actuelle, les groupements d'achats émettent des appels d'offres pour des termes de trois à cinq ans, qui permettent au fournisseur unique retenu de réduire ses coûts de production et de soumettre une offre inférieure pour le prochain appel d'offres. Prenons l'exemple de trois fabricants d'un produit injectable donné, qu’on appellera le médicament X. Les soumissions présentées en réponse à l'appel d'offres sont étudiées et le contrat est accordé au plus bas soumissionnaire, le fournisseur Y. Lors du prochain appel d'offres, le fournisseur Y a déjà en place une chaîne de fabrication rodée pour le médicament $X$, ce qui lui permet alors de soumissionner un prix plus bas que les deux autres fournisseurs. Avec le temps, le fournisseur $\mathrm{Y}$ pourrait obtenir des contrats avec les trois principaux groupements d'achats. Il n'est alors presque assurément plus rentable pour les autres fournisseurs de continuer à fabriquer le médicament $\mathrm{X}$, parce que leurs coûts de production sont trop élevés, et ils mettront ainsi probablement fin à la fabrication du médicament $\mathrm{X}$. Cette façon de tenter de réduire les coûts des médicaments dans le budget de l'hôpital entraîne une diminution de la concurrence et crée une situation dans laquelle le fournisseur $Y$ est l'unique fabricant du médicament X. Si la fabrication du médicament $X$ par ce fournisseur est " interrompue " pour une raison ou une autre, nous avons alors de gros problèmes. En fait, c'est ce qui se passe à l'heure actuelle!

Quelle leçon pouvons-nous tirer des pénuries actuelles de médicaments? La réponse est simple : nous devons maintenir une saine concurrence dans le marché canadien des médicaments afin de garantir la chaîne d'approvisionnement en médicaments essentiels. Ce n'est pas un concept nouveau. Par exemple, le groupement d'achats de vaccins pour les gouvernements fédéral, provinciaux et territoriaux avait bien 
compris le risque il y a des années. Ce groupement d'achats est responsable de l'approvisionnement en vaccins à l'échelle nationale. Afin de maintenir la capacité de production canadienne de vaccins contre la grippe, le groupement procède à un fractionnement du contrat depuis plusieurs années, octroyant la plus grande part du contrat au plus bas soumissionnaire et le reste au second et possiblement au troisième soumissionnaire le plus bas. Ce fractionnement peut être de 50\%-25\%-25\% s'il y a trois fournisseurs ou de $70 \%-30 \%$ s'il y en a seulement deux. Cette façon de faire garantit que plus d'un fabricant est en mesure de maintenir la chaîne de production. Par contraste, la Nouvelle-Zélande n'avait qu'un fournisseur de vaccin contre la grippe, Sanofi-Pasteur. En 2005, on a découvert que l'une des souches du virus grippal avait été sous-dosée dans le vaccin, ce qui signifiait que le gouvernement devait faire des pieds et des mains pour trouver un autre fabricant juste avant la saison de la grippe. S’il avait fractionné le contrat, le problème et les conséquences qui ont découlé de la rupture d'approvisionnement auraient pu être évités 5 .

La pratique du fractionnement des contrats n'est pas courante, mais certains groupements d'achats évaluent actuellement cette possibilité. Par exemple, le Comité de pharmacie de Medbuy a répertorié 20 médicaments injectables essentiels dont l'approvisionnement n'est assuré que par deux ou 3 fournisseurs au Canada. De ces 20 médicaments, 5 feront l'objet d'un fractionnement de contrat. Cette façon de faire exige la collaboration des fournisseurs et des hôpitaux. Les fournisseurs doivent être prêts à présenter une offre concurrentielle, même s'ils n'obtiennent pas la totalité du contrat, et les hôpitaux doivent être prêts pour leur part à payer plus pour une fraction du contrat. Même si nous essayons de réduire les coûts, nous devons favoriser un environnement propice à une saine concurrence et le fractionnement de contrat est une façon d'y parvenir.

En 2008, le gouvernement canadien avait été informé du risque de rupture d'approvisionnement en médicaments pour la population canadienne et du danger inhérent à la diminution de la concurrence entre les fabricants de médicaments génériques ${ }^{6}$. Avec la crise actuelle sur la scène publique, voilà l'occasion parfaite de veiller à informer le gouvernement des démarches qui peuvent être mises de l'avant pour améliorer l'approvisionnement en médicaments.

Pendant que nous attendons une solution plus globale aux pénuries de médicaments, nous devons convaincre les administrateurs de nos hôpitaux et nos gouvernements d'envisager des méthodes d'approvisionnement novatrices qui maintiendront une saine concurrence dans le marché des médicaments et nous permettront de prendre soin de nos patients.

[Traduction par l'éditeur]

\section{References}

1. Canadian drug shortages survey: final report. Ottawa $(\mathrm{ON})$ : Canadian Pharmacists Association; 2010. Publié au www.pharmacists.ca/ cpha-ca/assets/File/cpha-on-the-issues/DrugShortagesReport.pdf. Consulté le 27 mars 2012.

2. Kaakeh R, Sweet BV, Reilly C, Bush C, DeLoach S, Higgins B, et al. Impact of drug shortages on U.S. health systems. Am J Health Syst Pharm 2011; 68(19):1811-1819.

3. Gray A, Manasse HR Jr. Shortages of medicines: a complex global challenge. Bull World Health Organ 2012;90(3):158-158A.

4. A review of FDA's approach to medical product shortages. Silver Spring (MD) : US Food and Drug Administration; 2011. Publié au www.fda.gov/ downloads/aboutfda/reportsmanualsforms/reports/ucm277755.pdf. Consulté le 23 mai 2012

5. MacKay P. Is PHARMAC's sole supply tendering policy harming the health of New Zealanders? J N Z Med Assoc 2005;118(1214):U1433. Publié au http://journal.nzma.org.nz/journal/118-1214/1433/. Consulté le 28 mars 2012.

6. Pour une concurrence avantageuse des médicaments génériques au Canada: Préparons l'avenir. Ottawa $(\mathrm{ON})$ : Bureau de la concurrence Canada; 2008 nov. Publié au www.bureaudelaconcurrence.gc.ca/eic/site/cb-bc.nsf/ vwapj/GenDrugStudy-Report-081125-fin-f.pdf/\$FILE/GenDrugStudyReport-081125-fin-f.pdf. Consulté le 25 mai 2012.

Régis Vaillancourt, , O.M.M., C.D., B. Pharm., Pharm. D., FCSHP, est directeur de pharmacie au Centre hospitalier pour enfants de l'est de I'Ontario, à Ottawa, en Ontario. II est aussi rédacteur adjoint du JCPH.

\section{Address correspondence to:}

D'Régis Vaillancourt

Centre hospitalier pour enfants de l'est de I'Ontario

401, chemin Smyth

Ottawa (ON) K1H 8L1

Courriel : Rvaillancourt@cheo.on.ca 